\title{
GEORGIA IN THE WORLD OF CORONOMICS: THREATS AND CHALLENGES
}

\author{
NANA ASLAMAZISHVILI \\ PhD in Economics, \\ Invited Professor at the \\ Tbilisi State University, Georgia \\ aslamazishvilinana@gmail.com
}

Abstract. So far, much has been written and discussed about the rapid variability and complex predictability of the global economy, and different countries have more or less successfully dealt with the challenges they face. However, the global threat posed by the world in the form of COVID-19 puts the methods and approaches to combating economic crises completely upside down. What should be the strategy and tactics of the countries to start recovering the economies as quickly as possible under such kind of circumstances? In such a situation, it should be the best way to "hurry up slowly," that is, to be thoroughly consistent so that short-term emergency measures do not harm long-term goals.

Given the conditions of coronomics and the lessons the world has learned from it at this stage, recovery of the economics, in the earlier sense of the term, should be completely ineffective. We are accustomed to the fact that in the post-crisis period, economic recovery in a sense implies a more or less back-off to what was before the crisis. Given that this time a completely different "crisis" is occurring, essential structural changes and important transformations in many areas of the economy are needed to overcome its consequences. One of the clear lessons that must to be learned from Coronomics is that returning to what was already before would not be the right course of action for the economies focused on the sustainable development.

Georgia is a small open economy, and the fate of such economies has already been decided in advance: they will not be able to influence the global economy, and their efforts must be directed to protect themselves from the negative effects of the ongoing processes in the world. What are the priorities for such countries on this path if traditional sectors are vulnerable to certain types of crises and fail to cope with the task of generating revenue in extreme situations that are necessary, on the one hand, to balance their demands and, on the other hand, to meet external obligations? This is the reality that Georgia has faced in the face of coronomics.

How did the Georgian economy meet the shock of COVID-19? 2019 will be a turning point in many years for assessing economic outcomes, not just in Georgia. What are the dynamics of macroeconomic indicators and do they give a positive signal according to the data of this period? This article deals with the external economic aspects of these indicators.

The current account deficit as of 2019 was $\$ 900.5$ million, or 5.1 percent of gross domestic product. Historically, this is the best indicator in the history of independent Georgia. On the other hand, historically, the countrys external liabilities, which amount to $\$ 34.5$ billion, are 1.9 times higher than Gross Domestic Product and 3.2 times higher than the countrys foreign financial assets; The countrys external debt was 1.1 times higher than GDP at the end of 2019, while imports accounted for 40.8 percent of total consumption (intermediate and final).

Thus, Georgiass positioning on the challenges of COVID-19 is completely unfavorable and critical.

This article aims to discuss the main aspects of the countryss foreign sector accounts, the profitable and deficient articles of the balance of payments that traditionally determine the state of the current account, and how vulnerable these items are to external factors and shocks. The focus on this issue is to explore the ways in which it is possible to reduce the degree of dependence of the countryss economy on foreign shocks and achieve external economic stability.

However, it should be noted that the format of the article is not sufficient for in-depth discussion of a number of causeand-effect issues, for the evaluation of perspectives, and for deeper and more substantiated reasoning. Therefore, it can be said that the paper forms the main postulates on the issues under consideration, which together and each of them deserves in-depth research, but not in terms of fragmentary time and content, but in a complex and permanent mode.

KEYWORDS: BALANCE OF PAYMENTS, CURRENT ACCOUNT, FOREIGN TRADE, INVESTMENTS, TRAVEL, COVID-19.

For citation: Aslamazishvili, N., (2020). Georgia in the World of Coronomics: Threats and Challenges. Globalization and Business, 10. 94-102. (In Georgian) https://doi.org/10.35945/gb.2020.10.012 


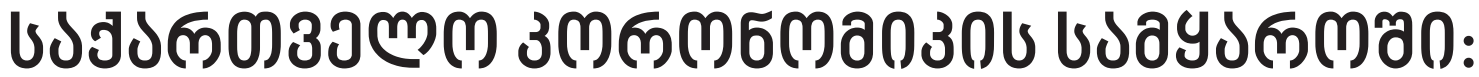

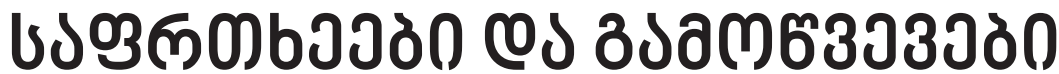

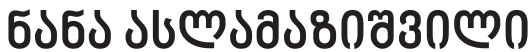

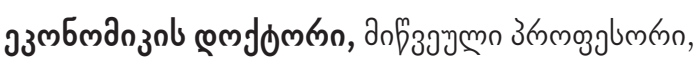

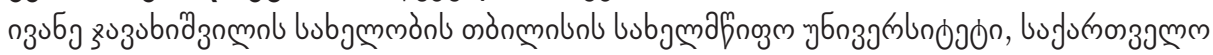

aslamazishvilinana@gmail.com

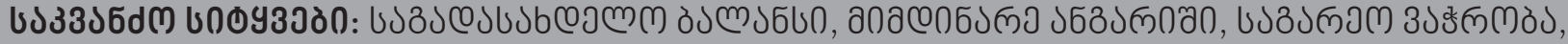

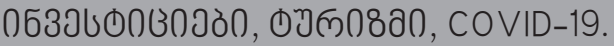

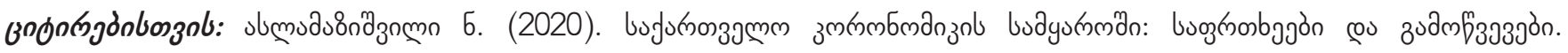

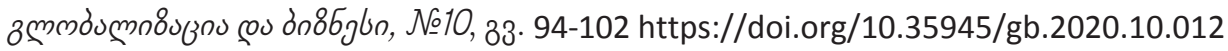

\section{ฆวเ১ว১ल0}

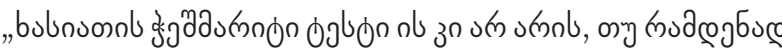

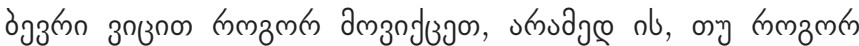

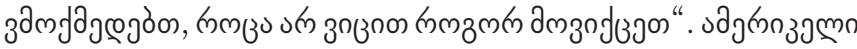

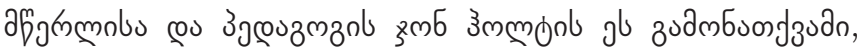

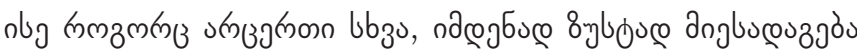

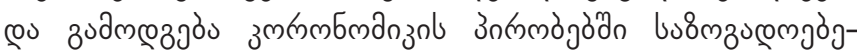

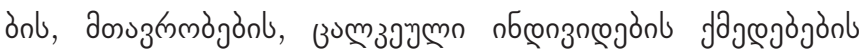
gaogubjòntonzol.

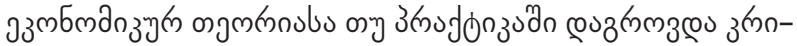

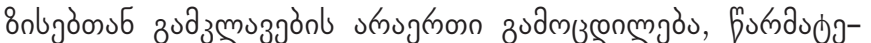

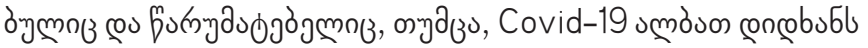

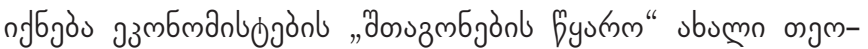

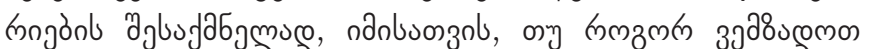

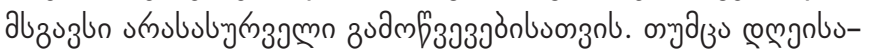

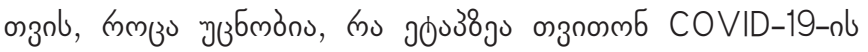

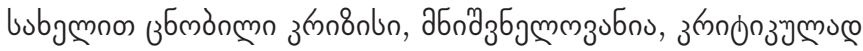

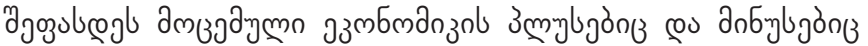

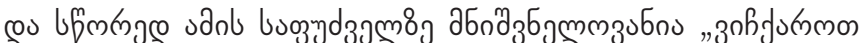

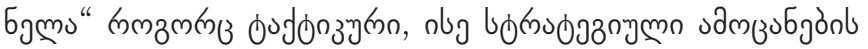

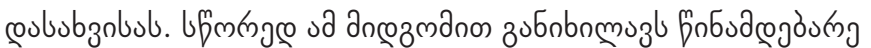

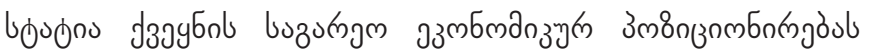

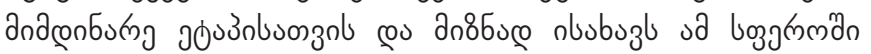

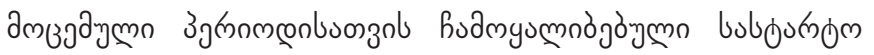

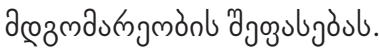

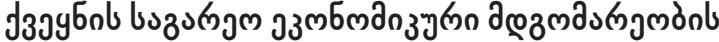

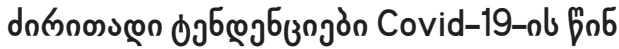

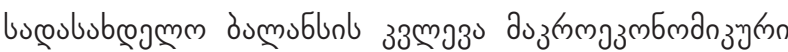

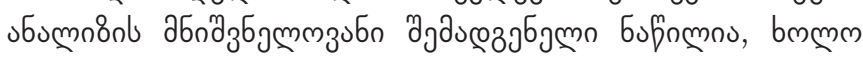

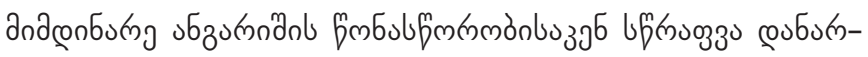

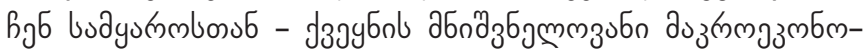

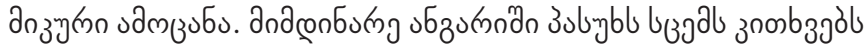

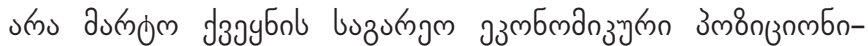

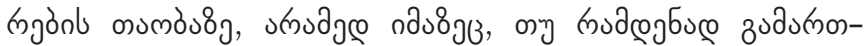

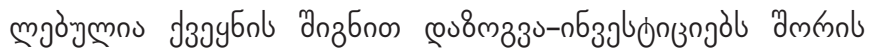

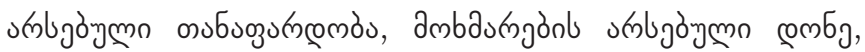

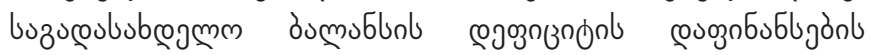

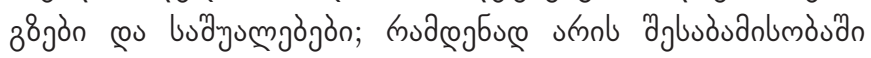

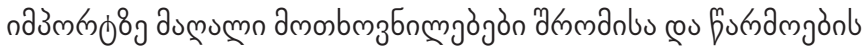

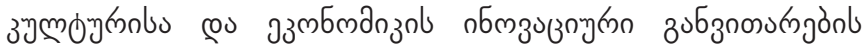

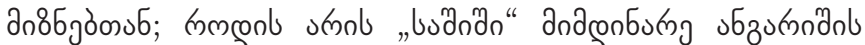

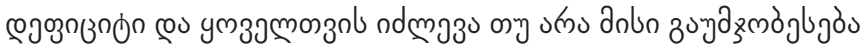

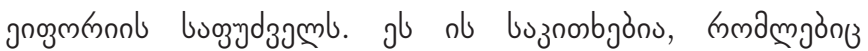

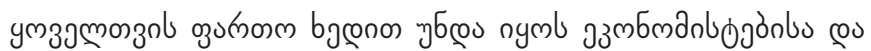

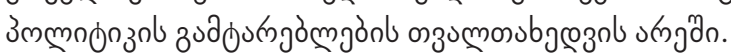

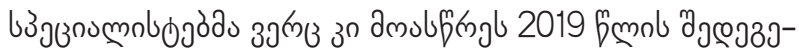

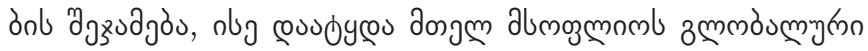

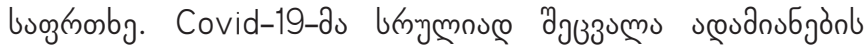

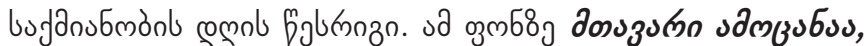

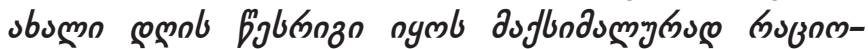

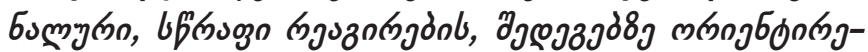

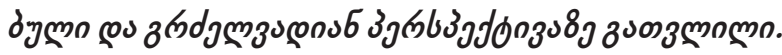

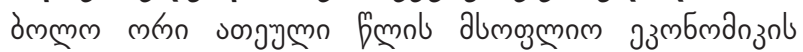

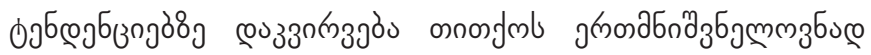

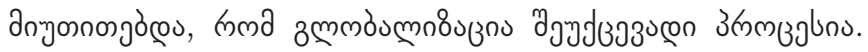

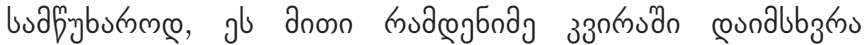

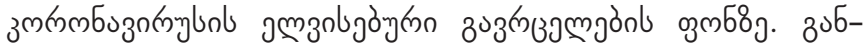

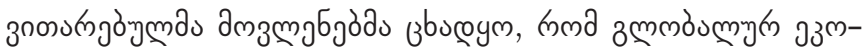

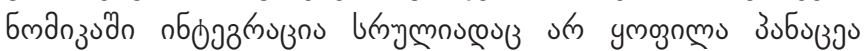

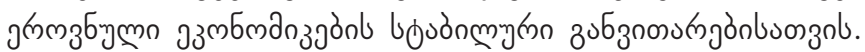

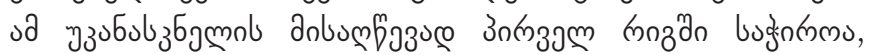




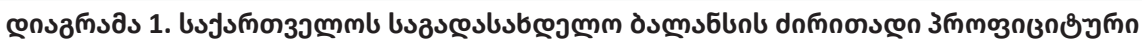

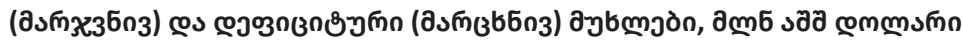

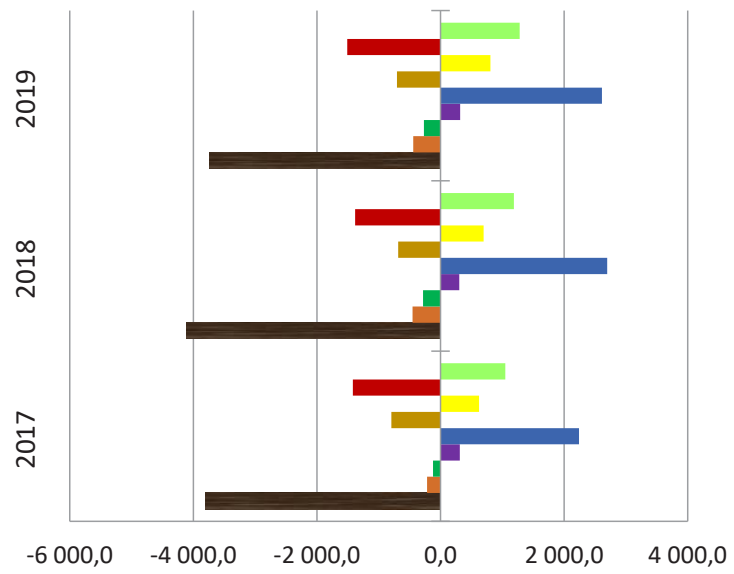

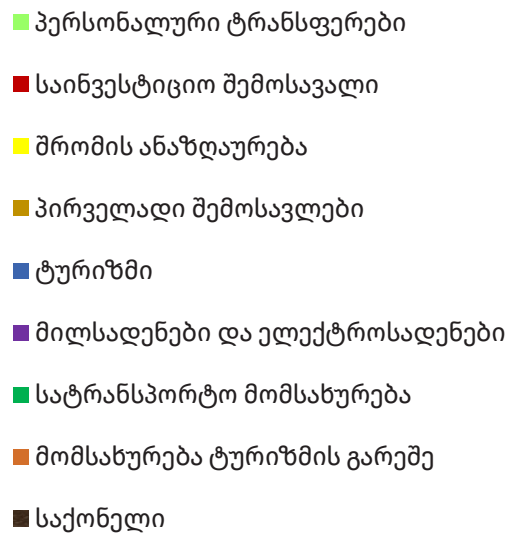

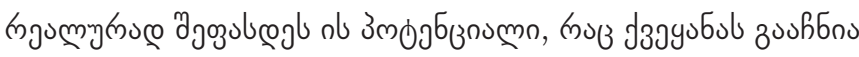

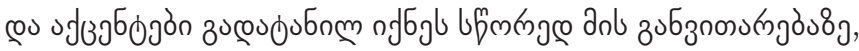

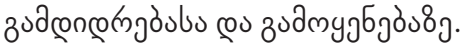

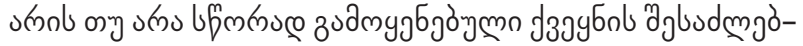

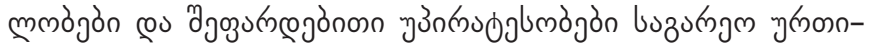

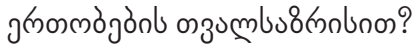

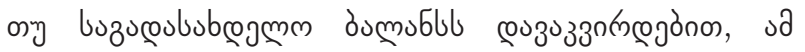

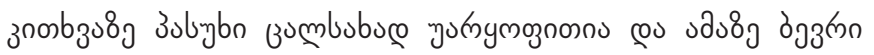

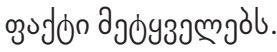

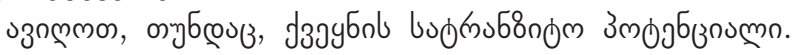

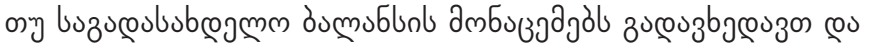

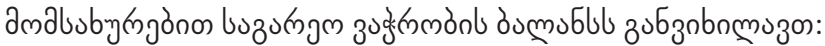

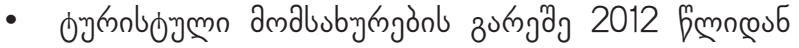

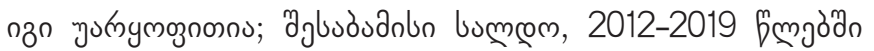

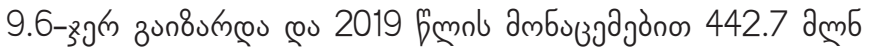

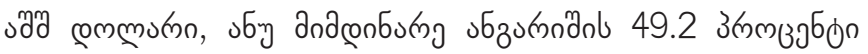

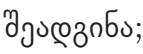

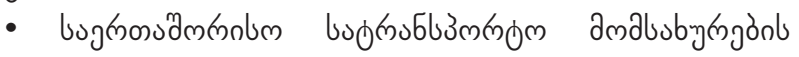

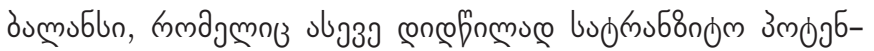

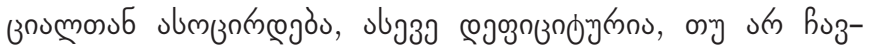

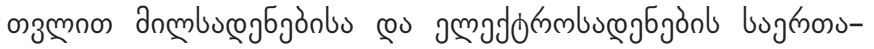

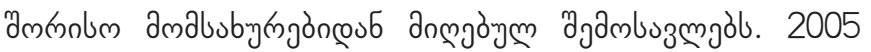

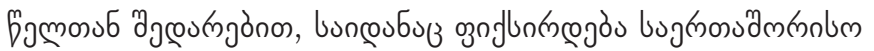

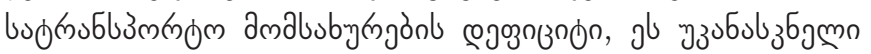

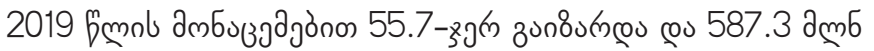

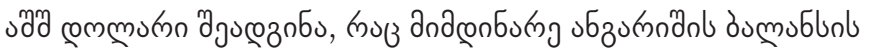
65.23 mmujóons.

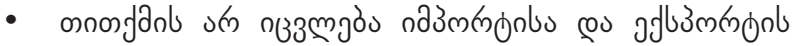

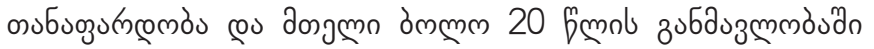

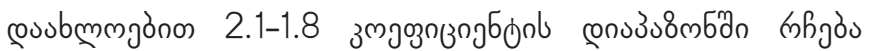

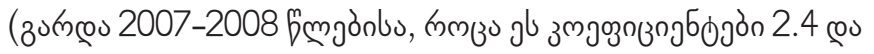

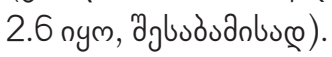

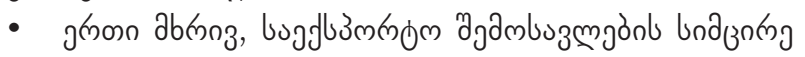

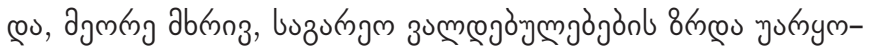

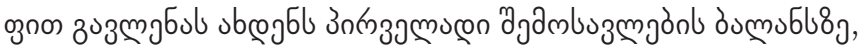

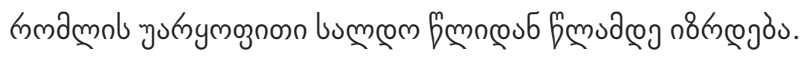

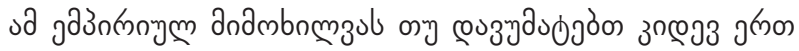

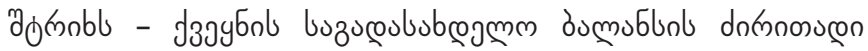

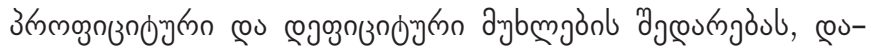

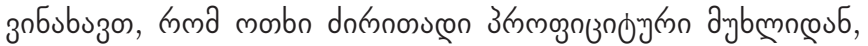

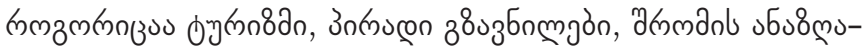

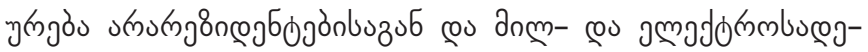

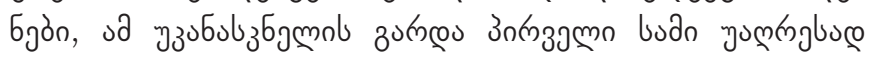

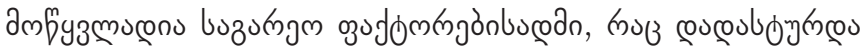

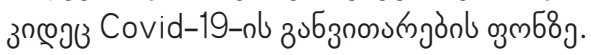

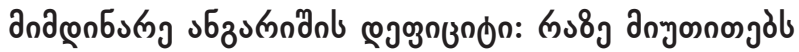

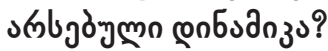

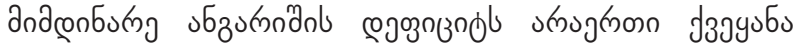

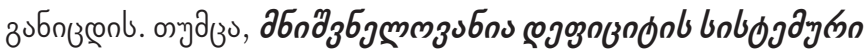

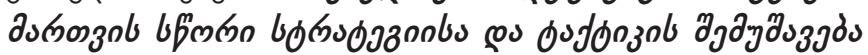
एu zubbmhrз

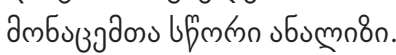

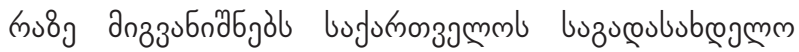
oumublinb loudonbozo?'

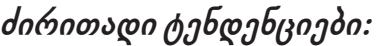

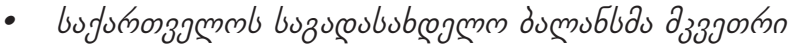

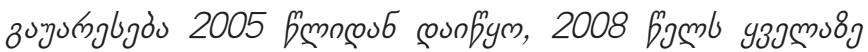

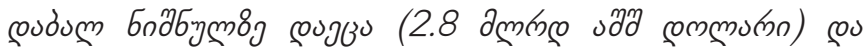

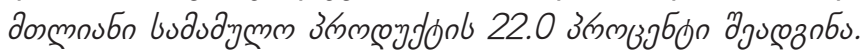

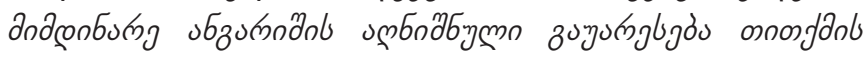

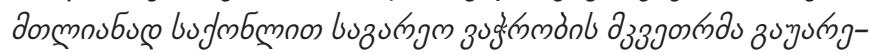

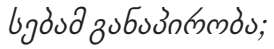

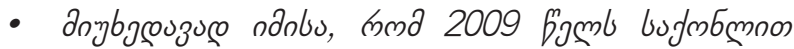

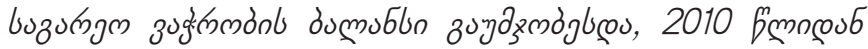

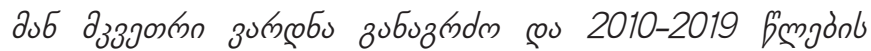

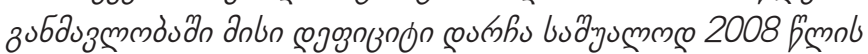

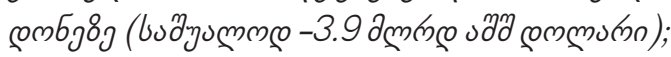

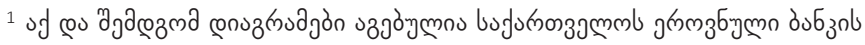

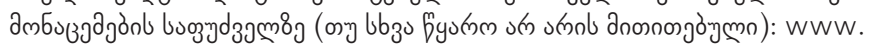
nbg.gov.ge. 


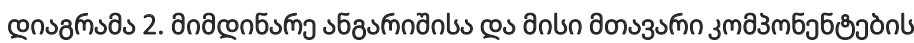

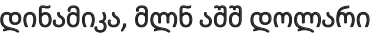

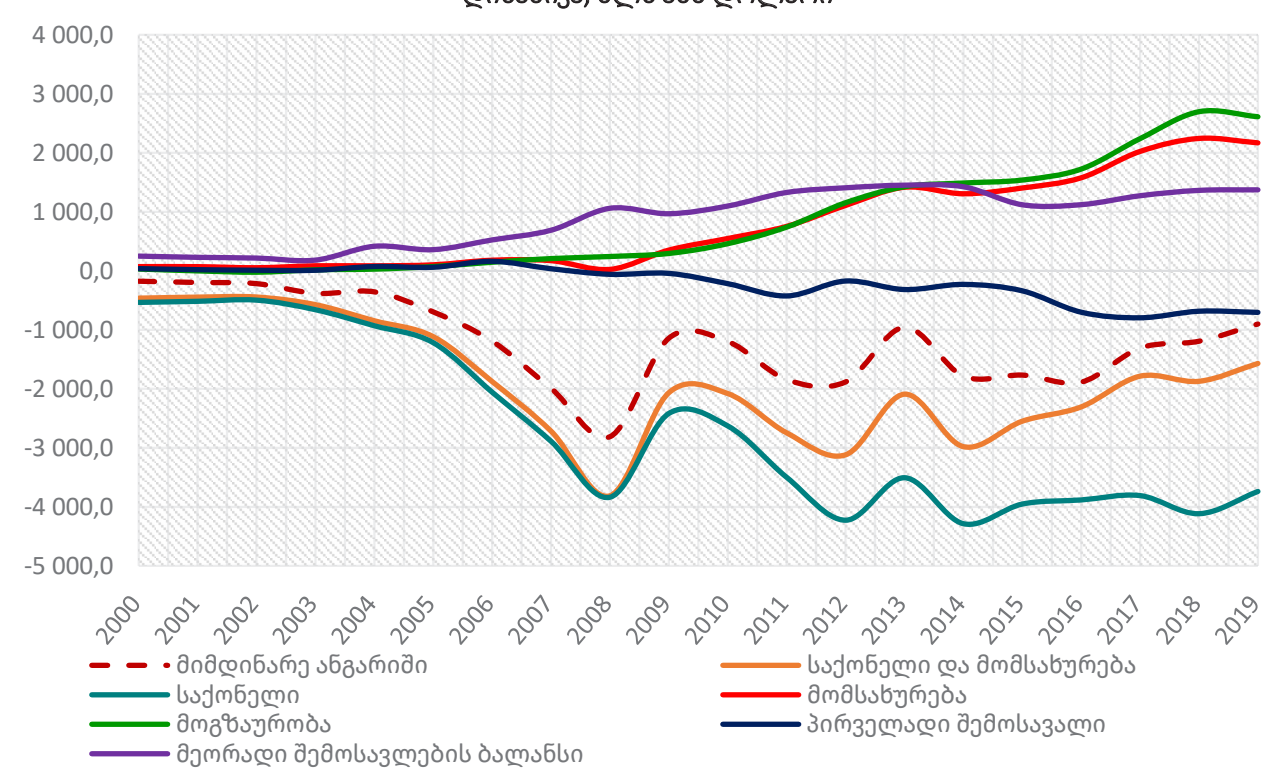

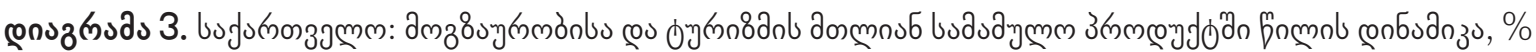

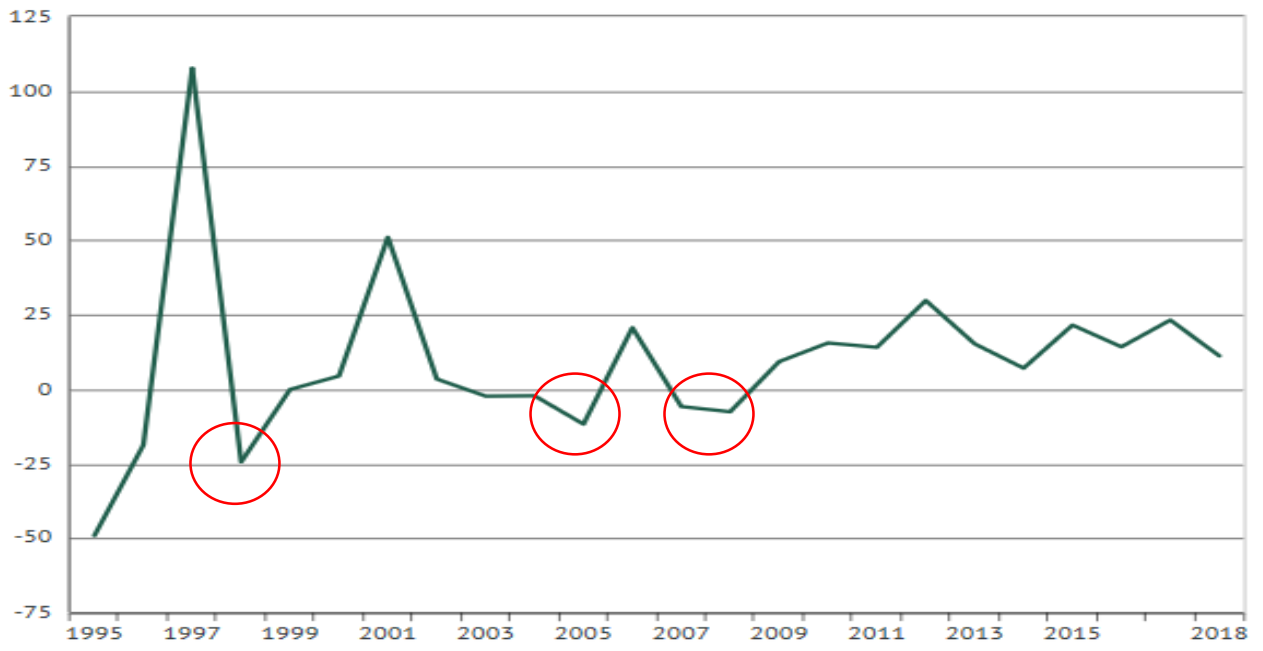

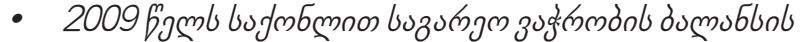
grnos:

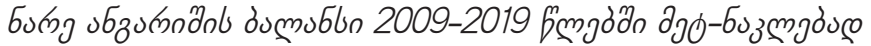

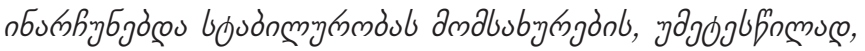

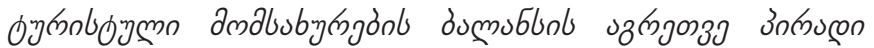

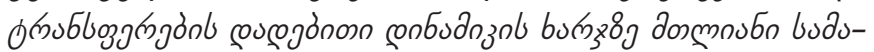

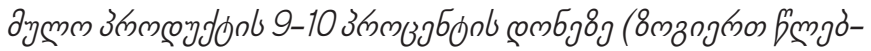

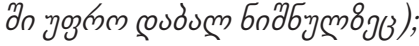

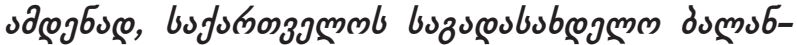

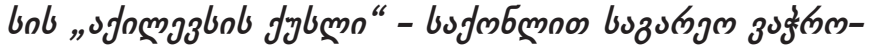

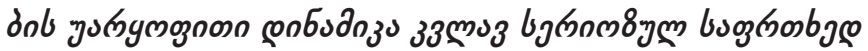

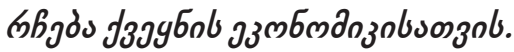

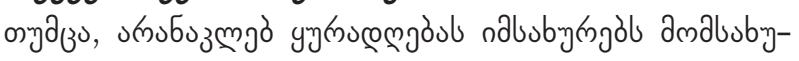

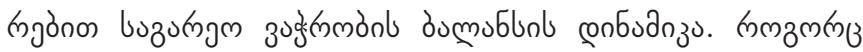

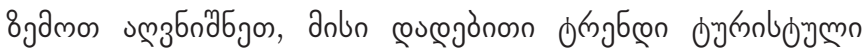

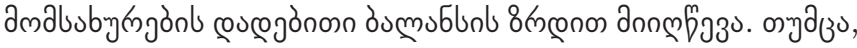

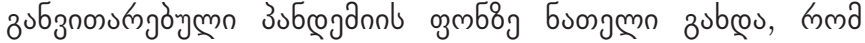

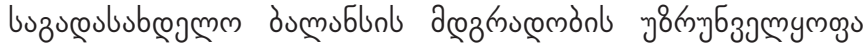

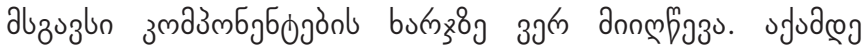

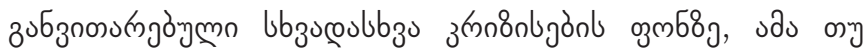

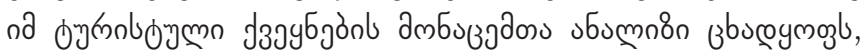

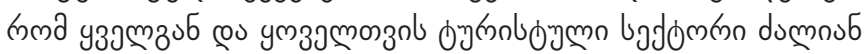

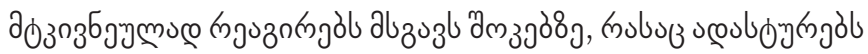

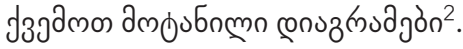

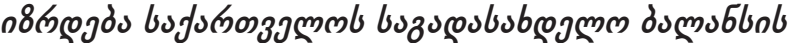

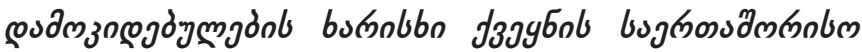

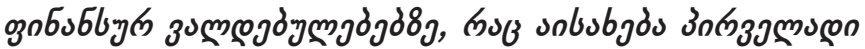

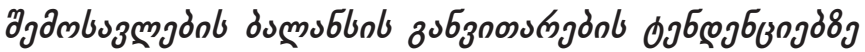

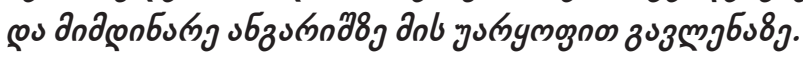

2 https://knoema.com/atlas/topics/Tourism/Travel-and-Tourism-TotalContribution-to-GDP/Contribution-of-travel-and-tourism-to-GDPgrowth?type=maps 


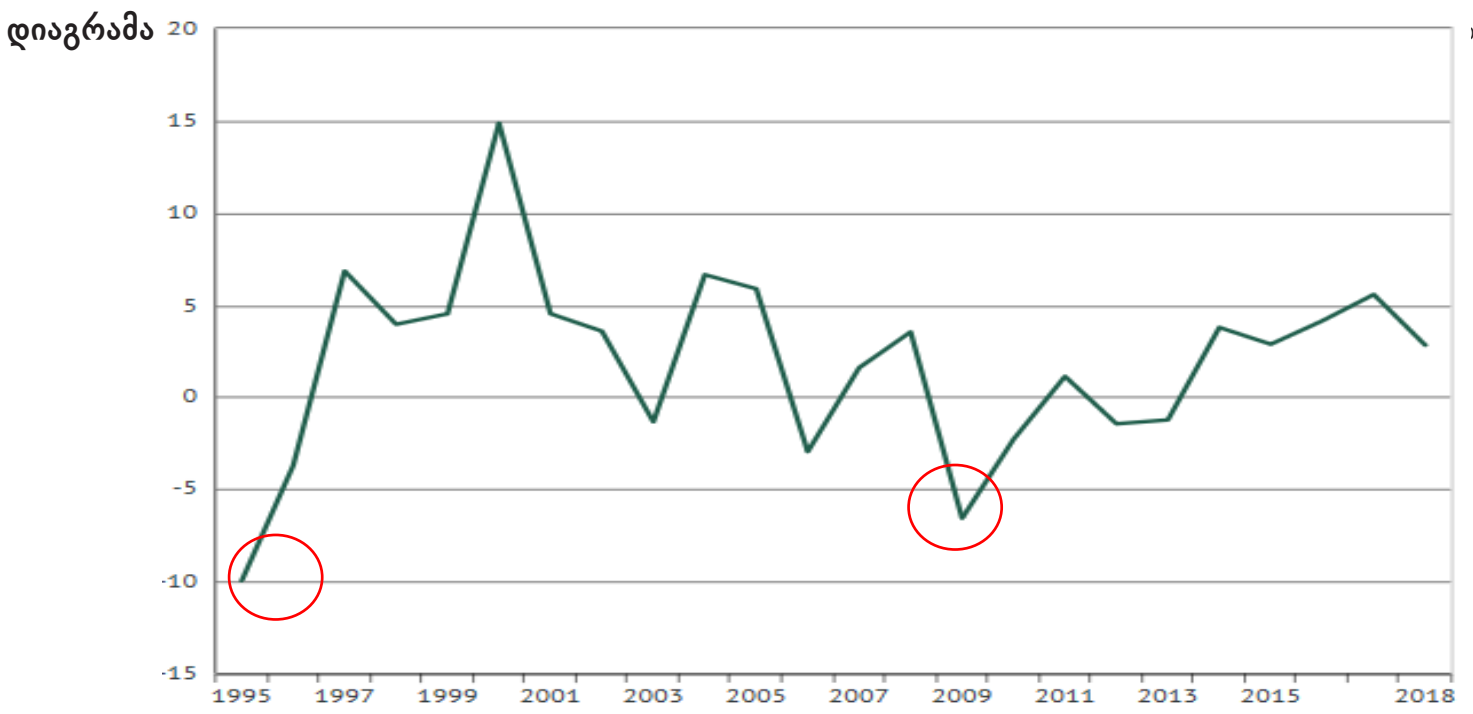

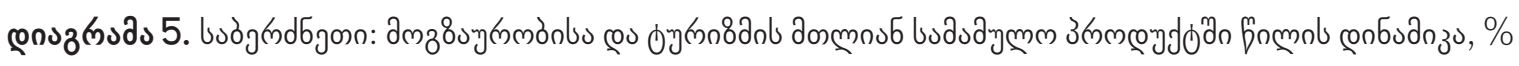

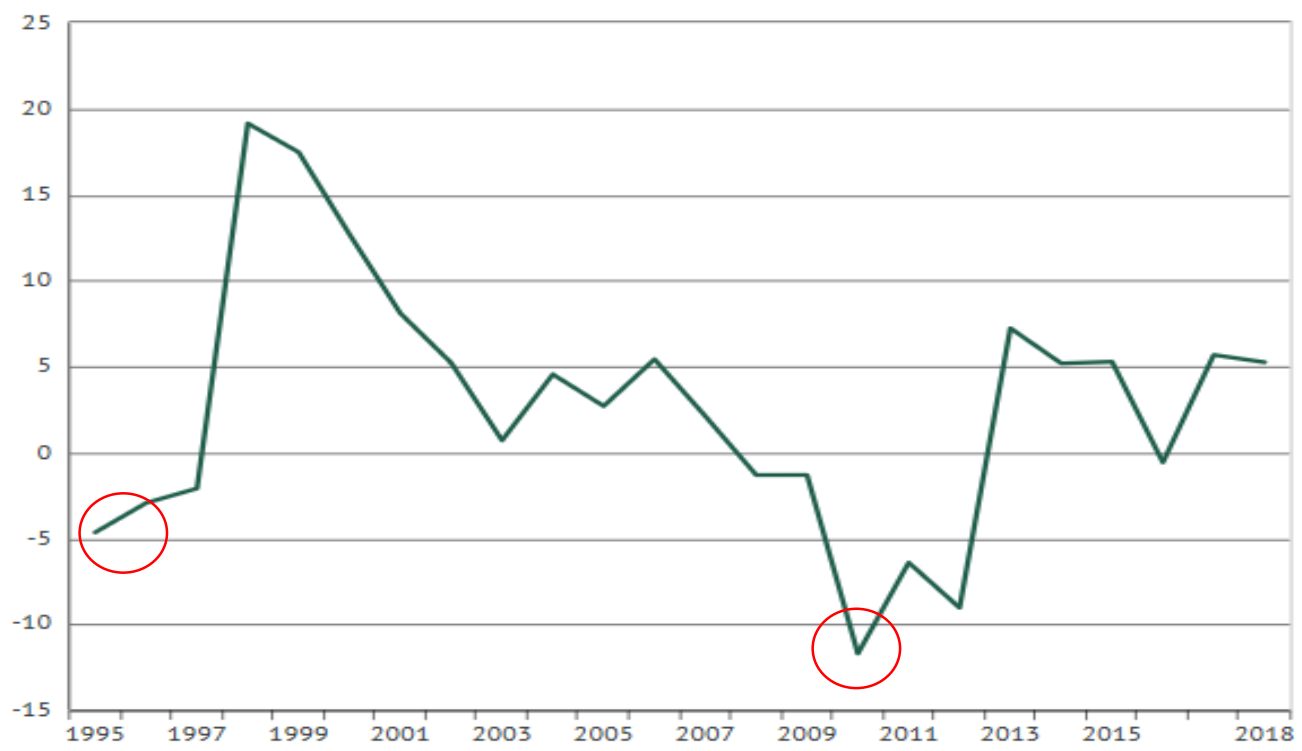

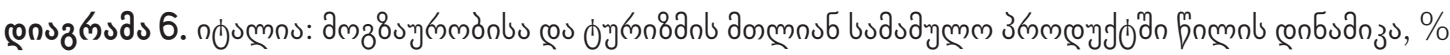

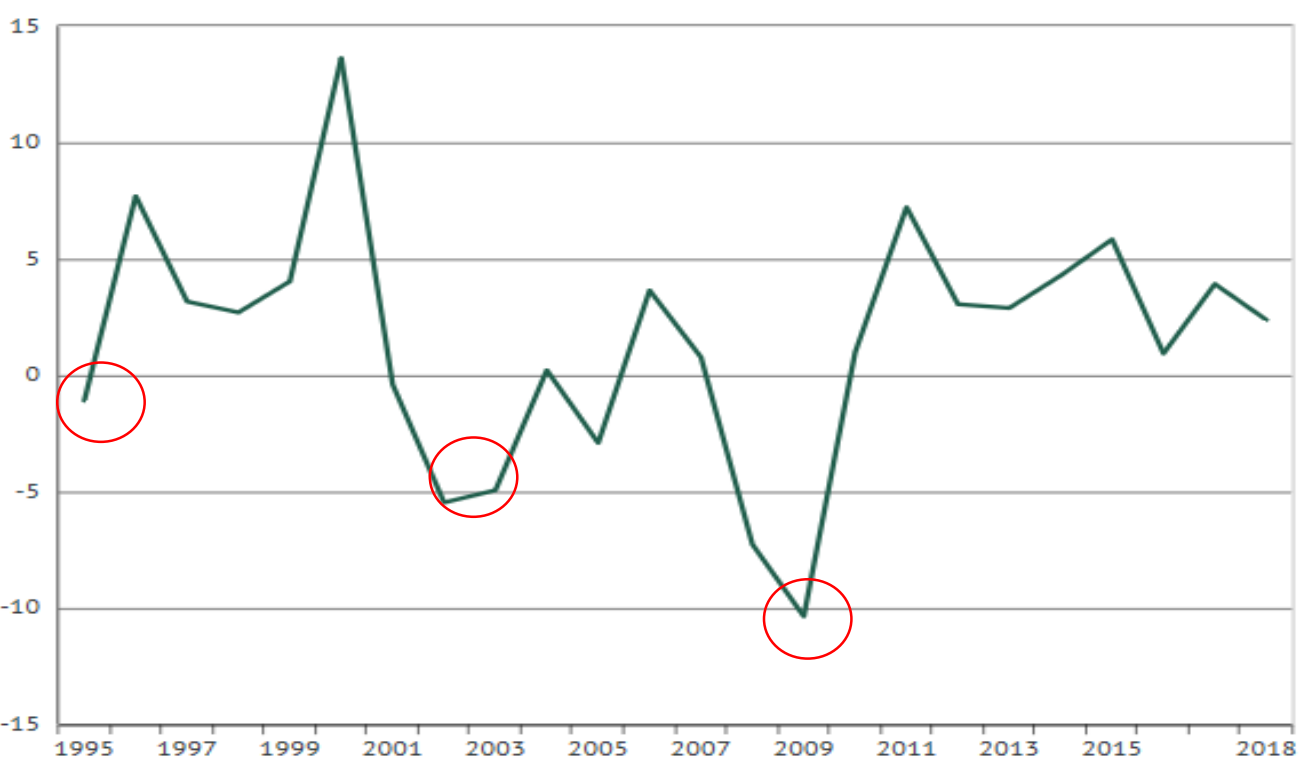




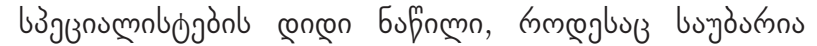

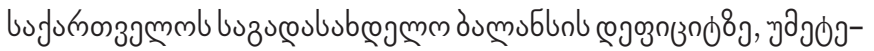

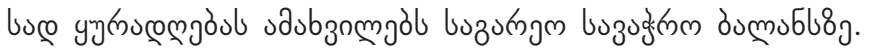

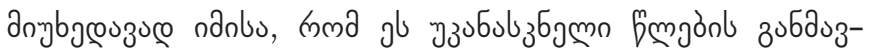

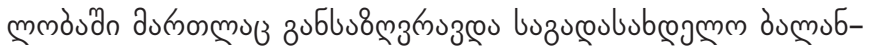

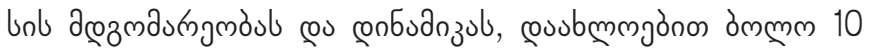

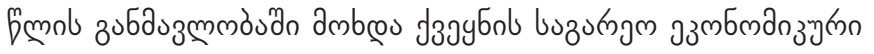

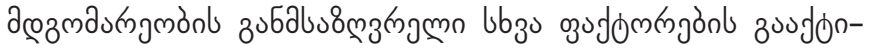

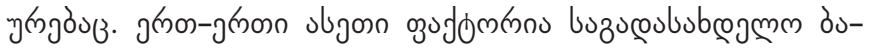

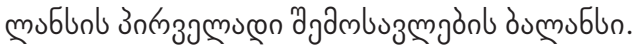

\section{domnosucon ojersjobajo:}

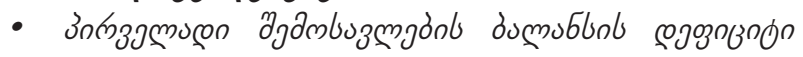

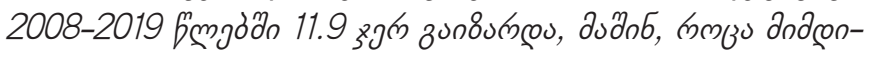

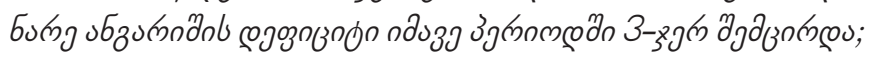

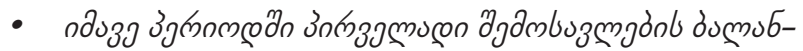

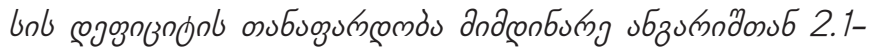

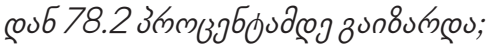

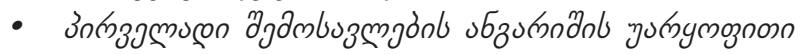

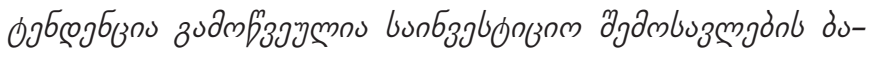

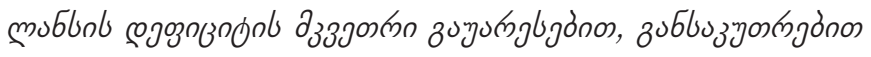

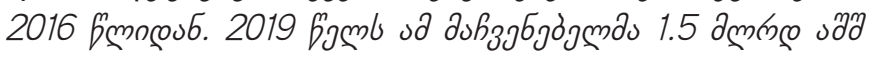

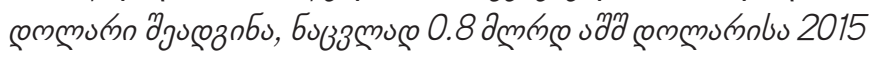
bogmb.

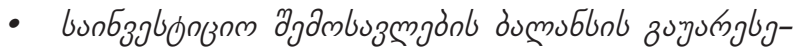

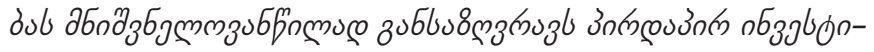

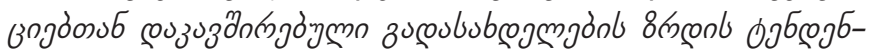

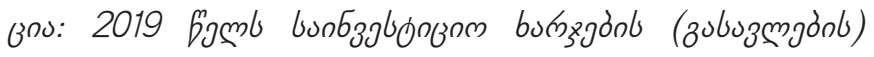

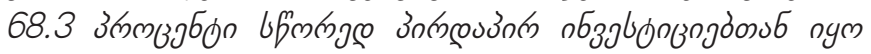

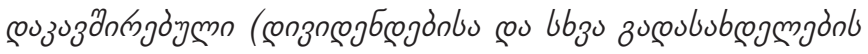

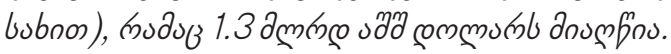

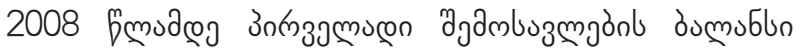

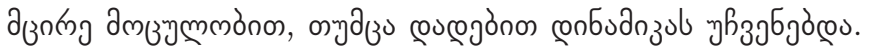

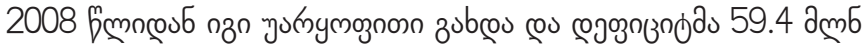

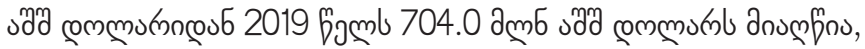

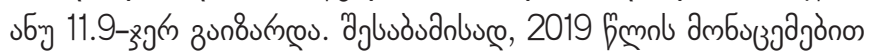

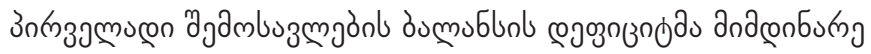

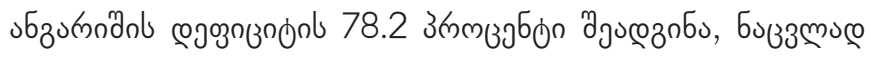
2.13 mmiзjбon 2008 bambl.

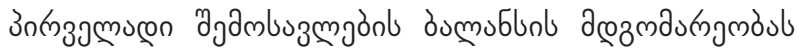

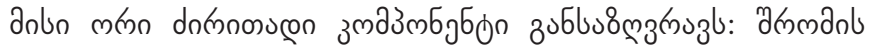
ง6u8

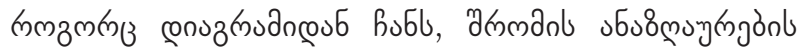

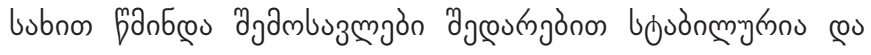

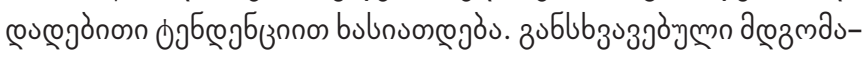

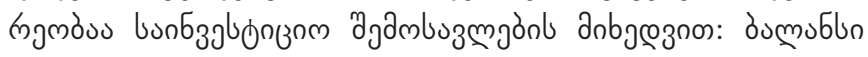

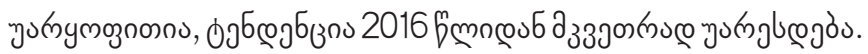

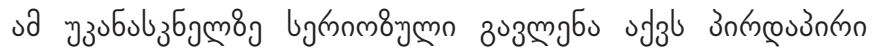

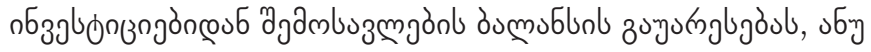

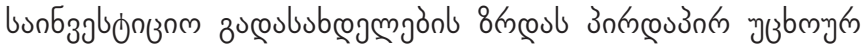

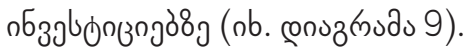

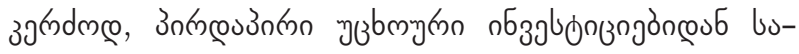

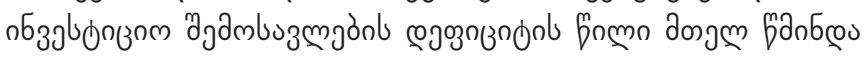

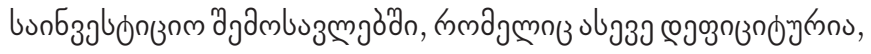

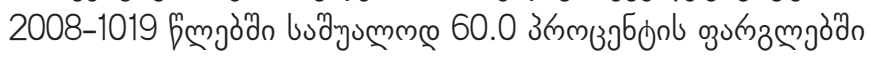

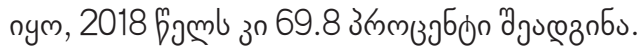

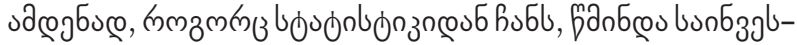

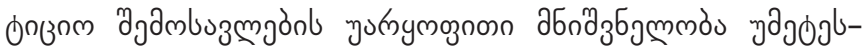

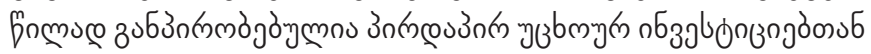

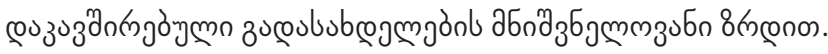

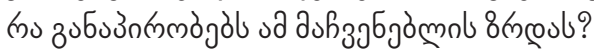

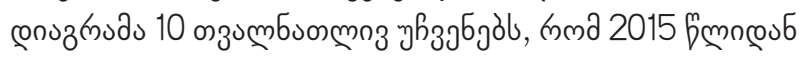

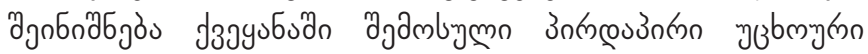

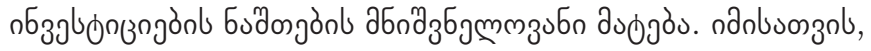

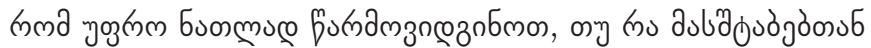

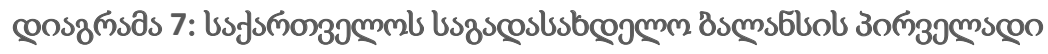

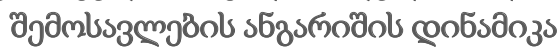

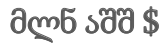

2000,00

1500,00

1000,00

500,00

0,00

$-500,00$

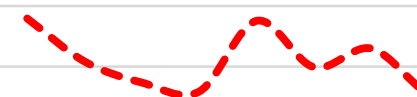

20,0

$-1000,00$

$-1500,00$

$-2000,00$

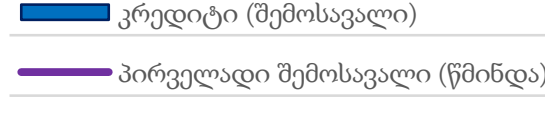

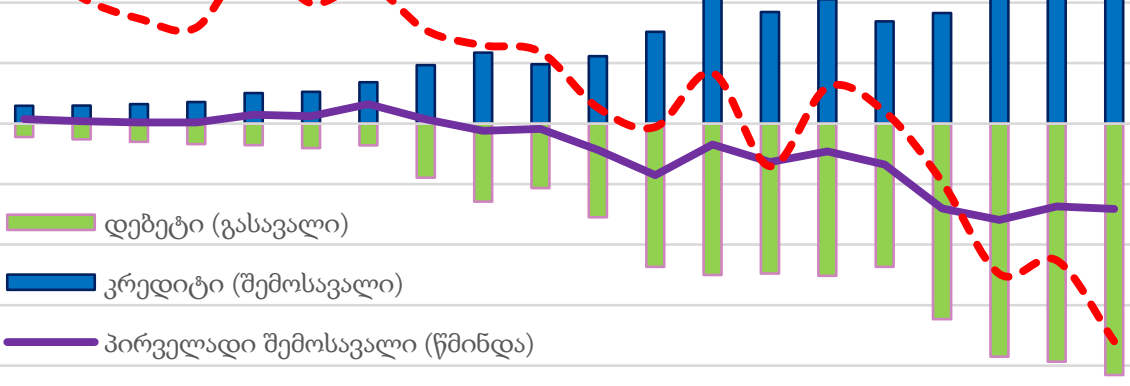

0,0

$-20,0$

$-40,0$

$-60,0$

$-80,0$

$-2500,00$

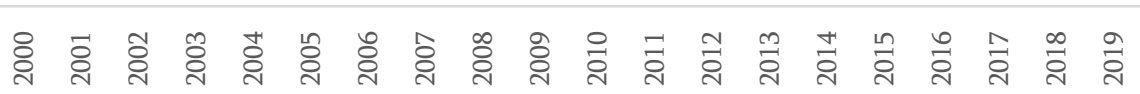




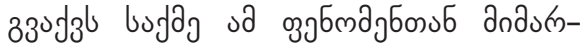

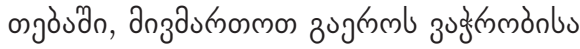

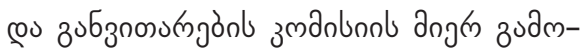

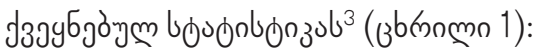

\section{งdeg6uณ:}

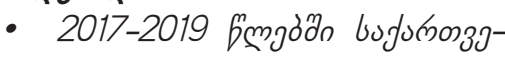

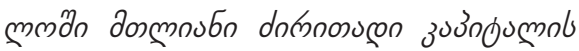

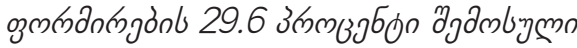

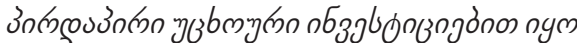

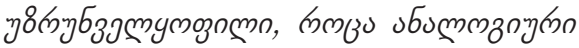

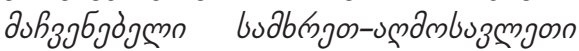

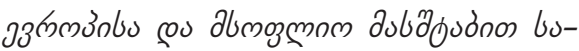

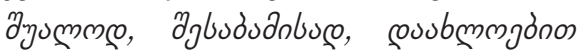

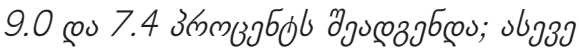

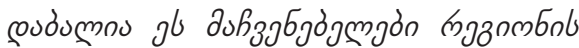

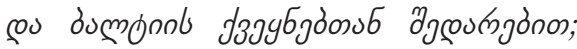

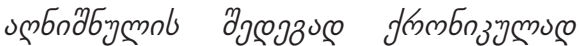

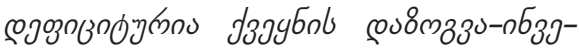

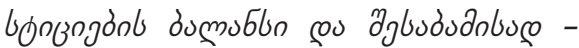
andsonбumg u busunan.

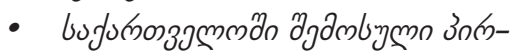

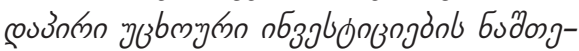

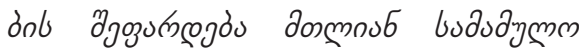

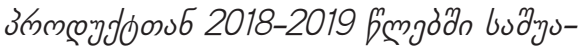

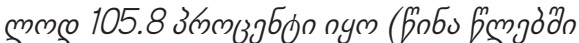

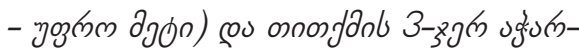

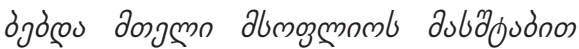

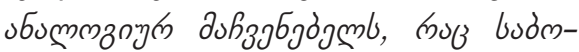

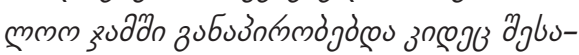

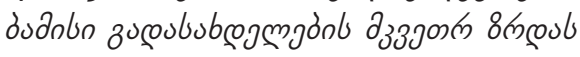

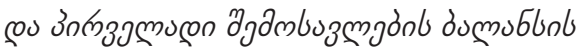
cojognogol.

bunbzglonzom zunoububegmgón

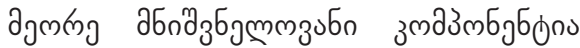

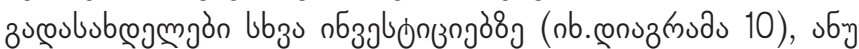

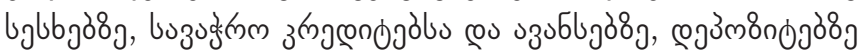

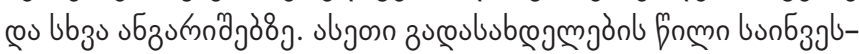

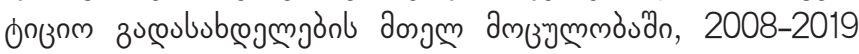

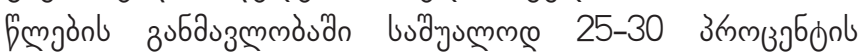

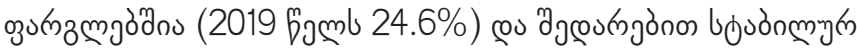

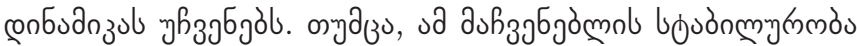

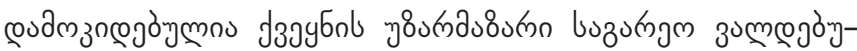

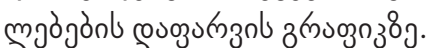

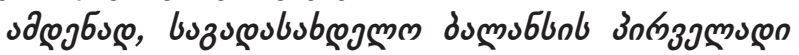

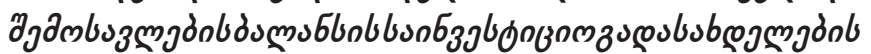

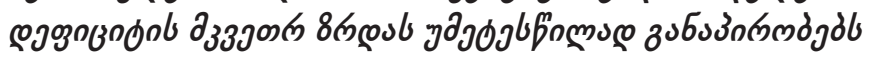

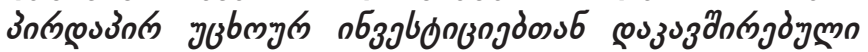
вucoububegrmgdo.

3 World Investment Report 2019. Country fact sheet: Georgia. https:// unctad.org/sections/dite_dir/docs/wir2020/wir20_fs_ge_en.pdf

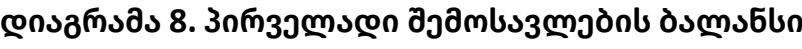

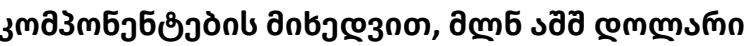

1000,00

500,00

0,00

$\begin{array}{llllllllllll}1 & 2 & 3 & 4 & 5 & 6 & 7 & 8 & 9 & 10 & 11 & 12\end{array}$

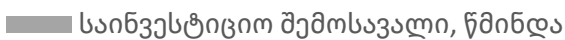

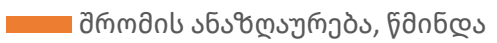

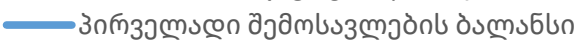

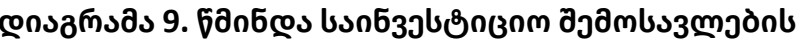

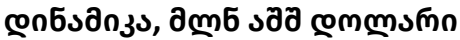

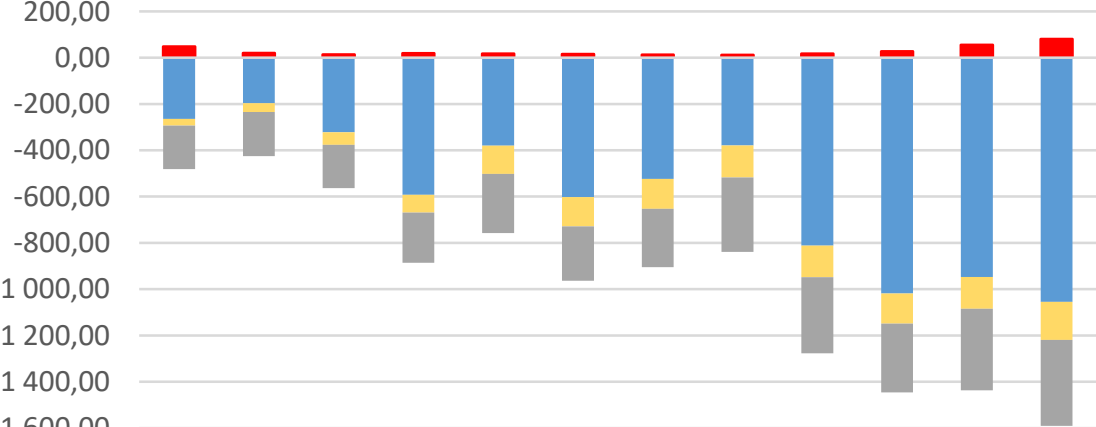

200820092010201120122013201420152016201720182019

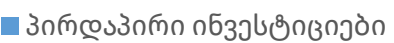

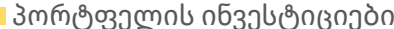

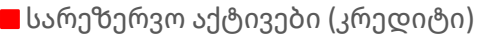

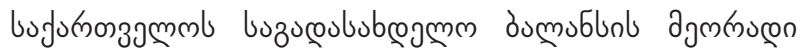

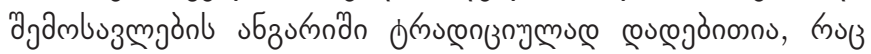

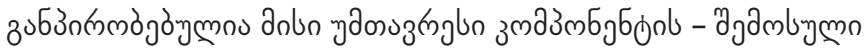

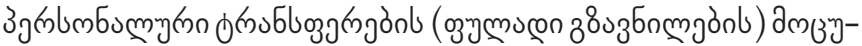

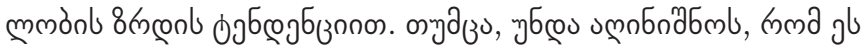

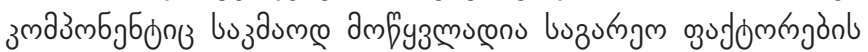
andurnon.

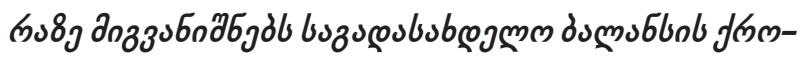

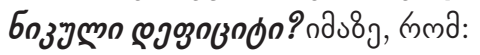

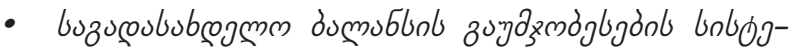

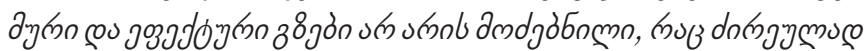

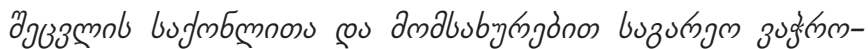

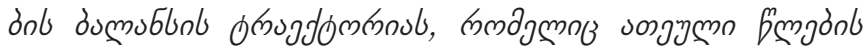

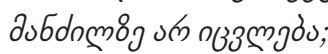

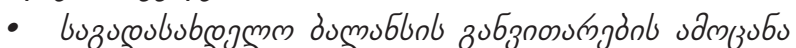

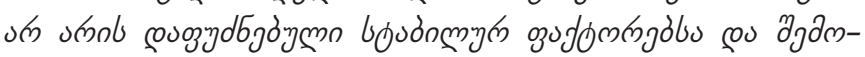




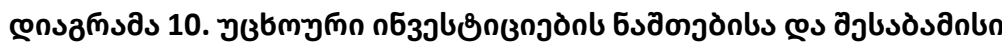

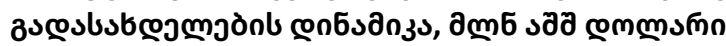

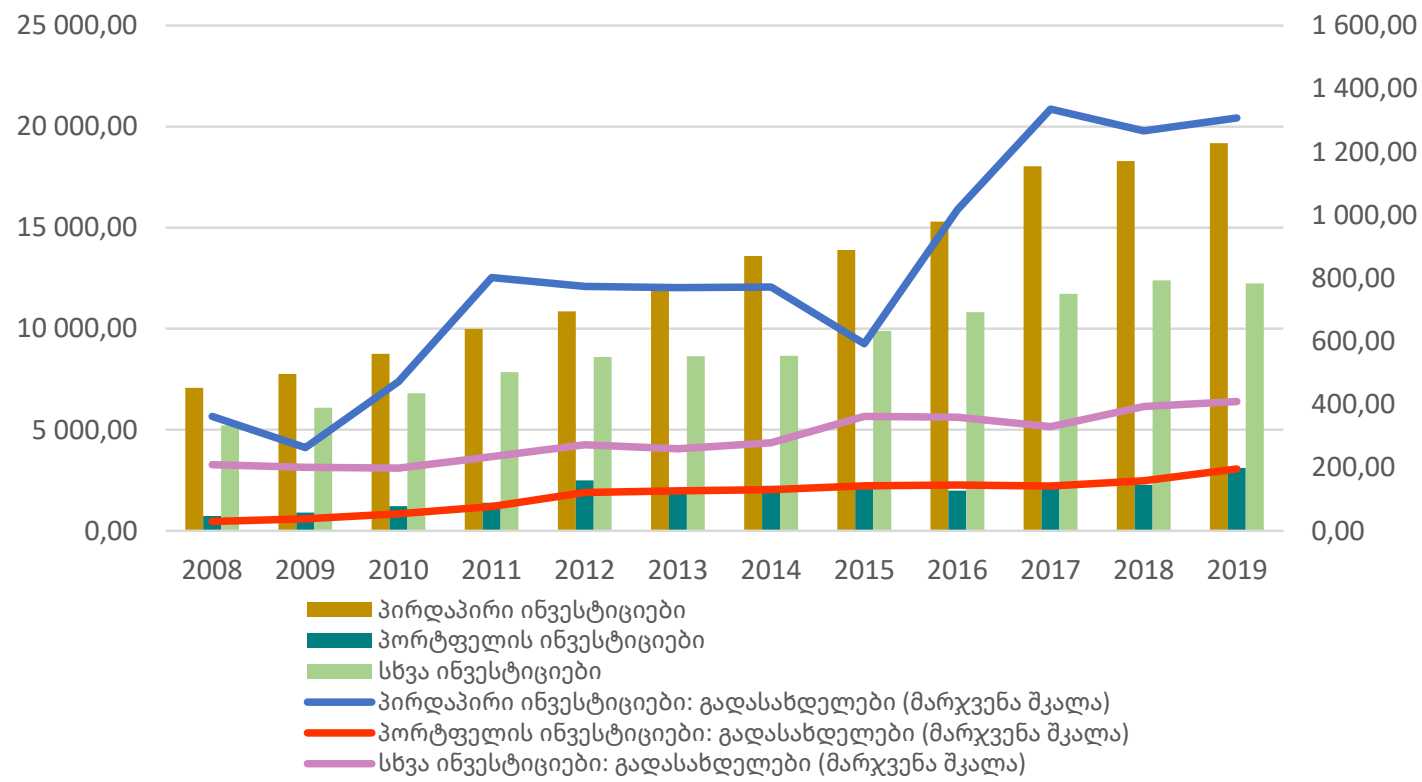

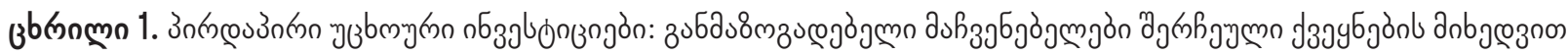

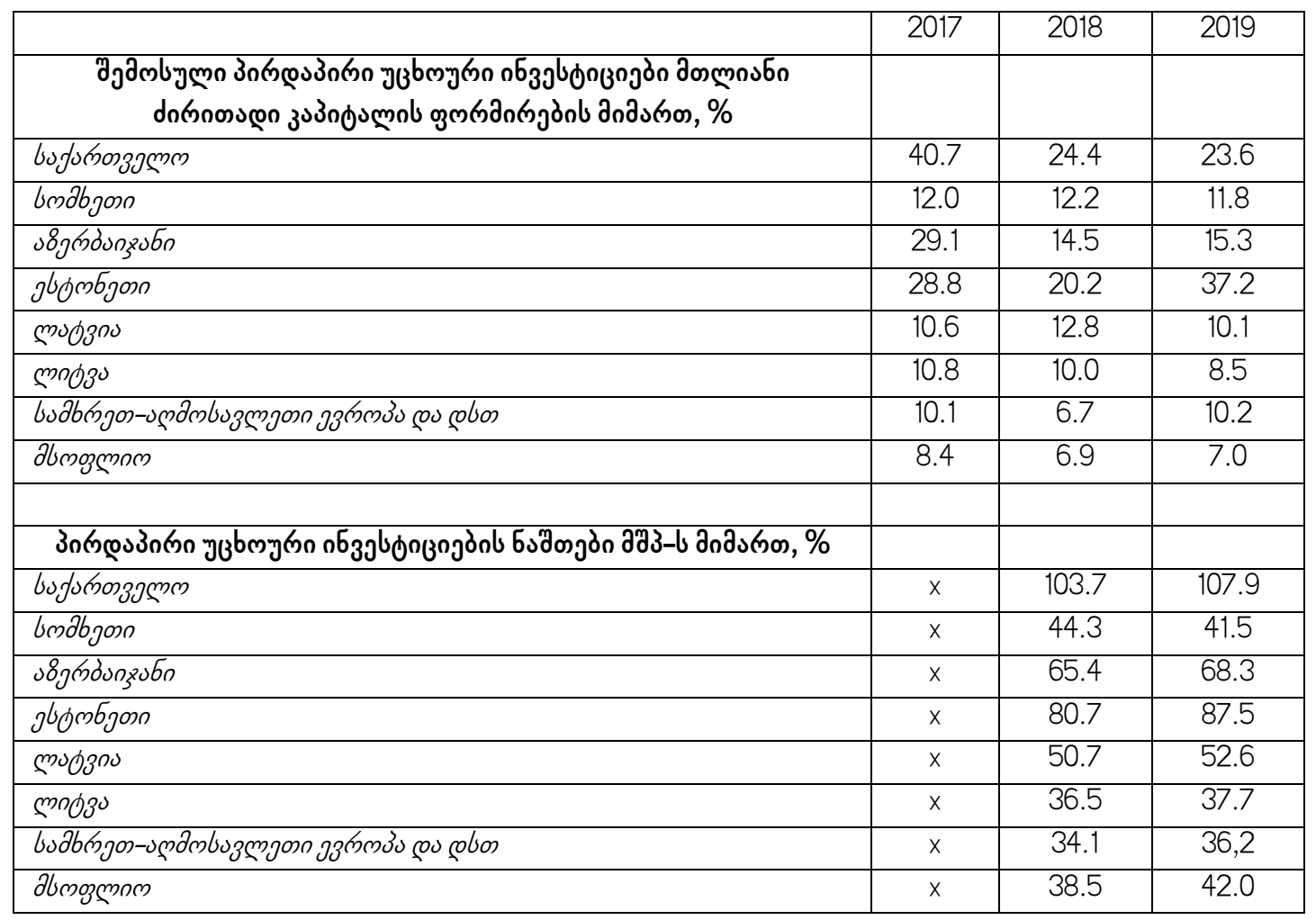

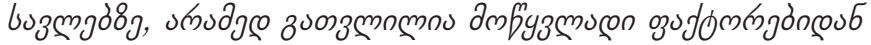

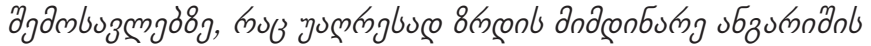

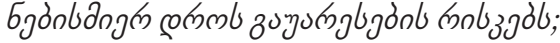

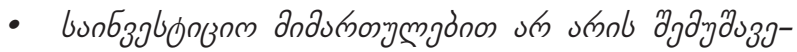

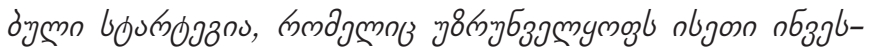

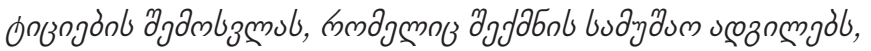
ubminn

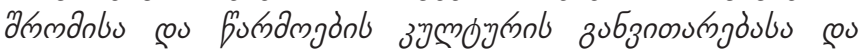

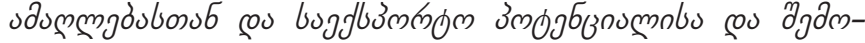

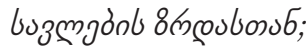

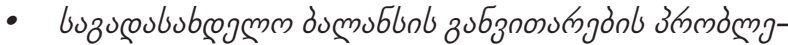

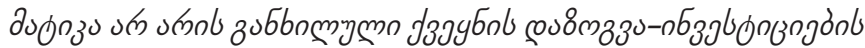

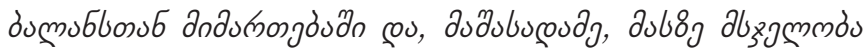

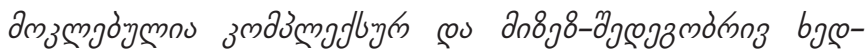

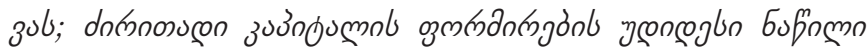

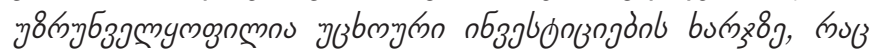




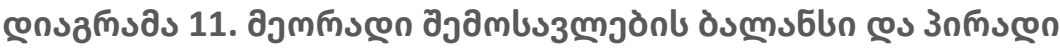

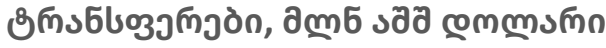

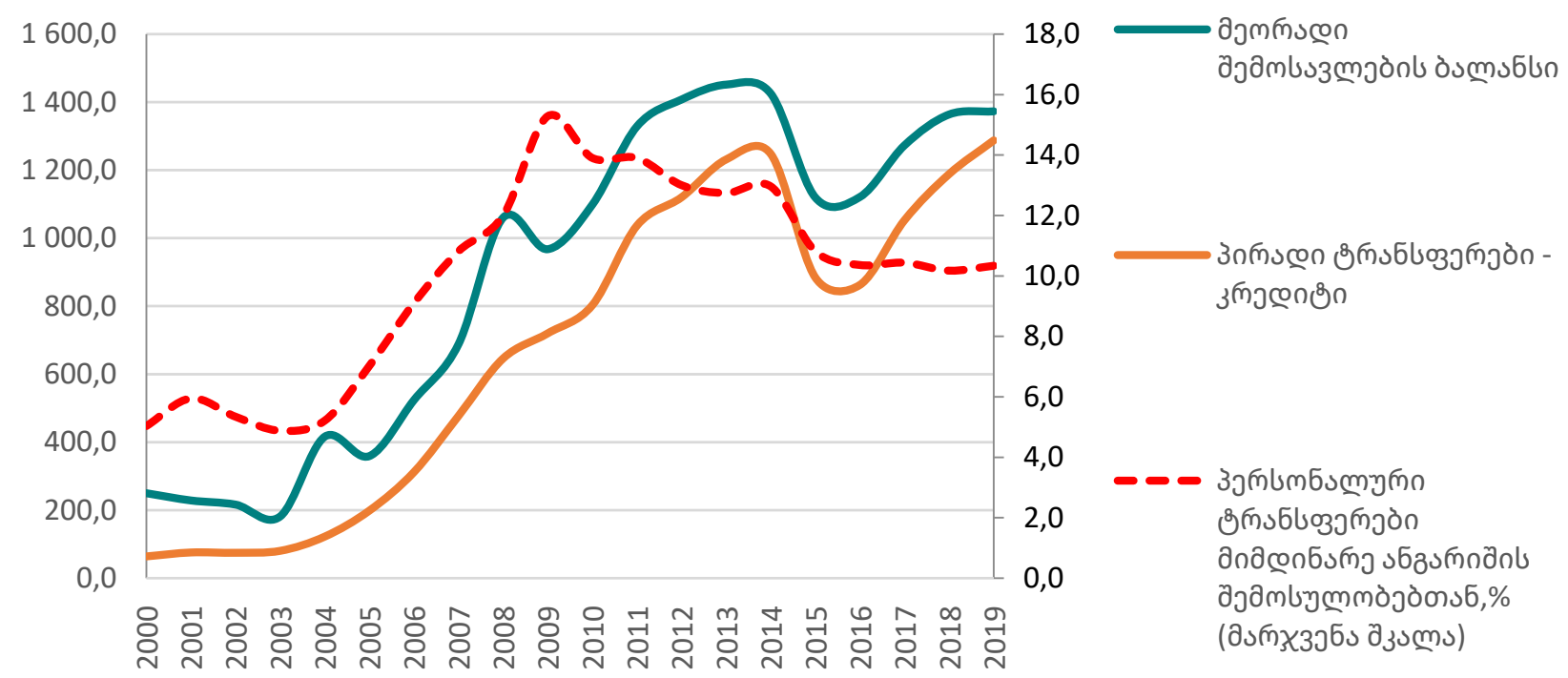

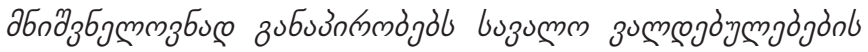

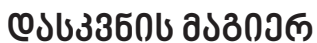

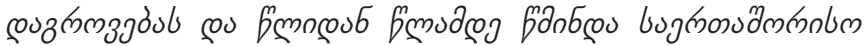

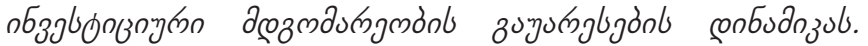

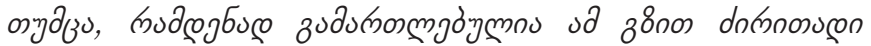

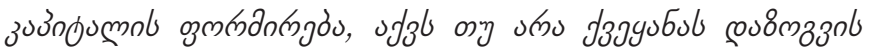

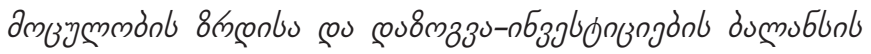

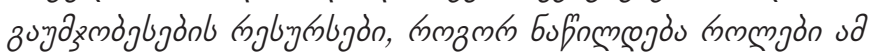

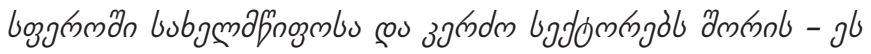

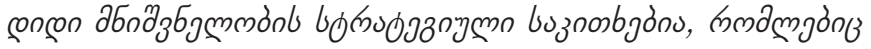

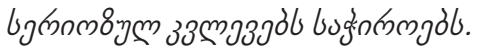

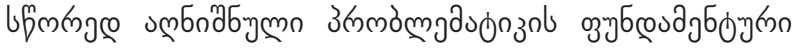

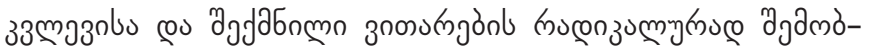

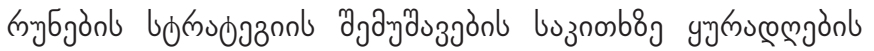

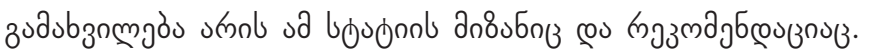

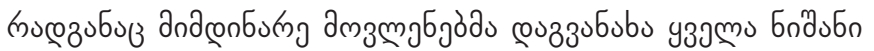

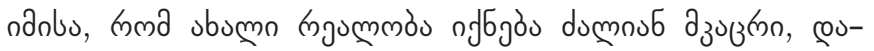

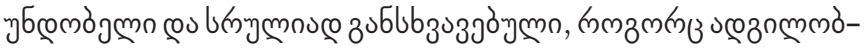

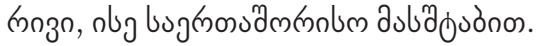

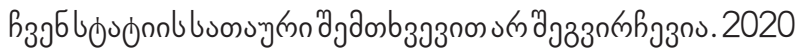

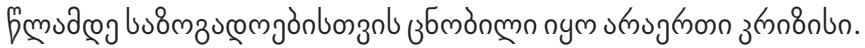

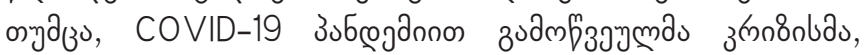

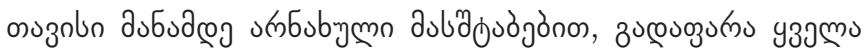

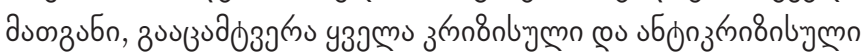

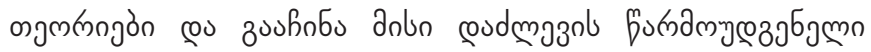

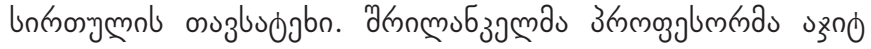

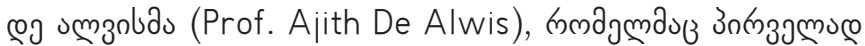

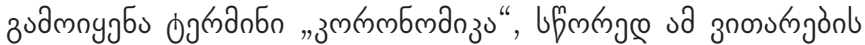

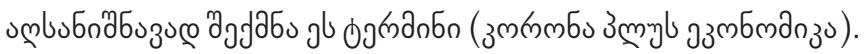

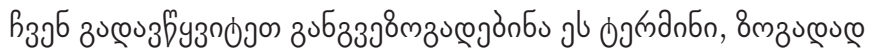

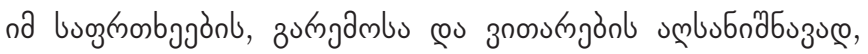

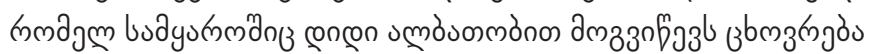

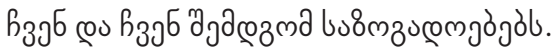

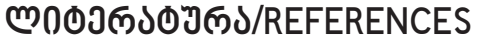

Aslamazishvili, N. (2018). Balance of Payments of Georgia: Key Aspects of its Analysis. Globalization \& Business N6. $136-143$. (In Georgian)

Balance of Payments of Georgia (2018). National Bank of Georgia. https://www.nbg.gov.ge/uploads/bop/bop_2018_eng_ publish.pdf

World Investment Report (2019). Country fact sheet: Georgia. https://unctad.org/sections/dite_dir/docs/wir2020/wir20_fs_ ge_en.pdf

https://knoema.com/atlas/topics/Tourism/Travel-and-Tourism-Total-Contribution-to-GDP/Contribution-of-travel-andtourism-to-GDP-growth?type=maps

www.nbg.gov.ge 